\title{
Pan-cancer analysis of KEAP1 mutations as biomarkers for immunotherapy outcomes
}

\author{
Xiaoxia Chen", Chunxia Su", Shengxiang Ren", Caicun Zhou, Tao Jiang \\ Department of Medical Oncology, Shanghai Pulmonary Hospital \& Thoracic Cancer Institute, Tongji University School of Medicine, Shanghai \\ 200433, China \\ \#These authors contributed equally to this work. \\ Correspondence to: Prof. Caicun Zhou, MD, PhD; Prof. Tao Jiang, MD, PhD. Department of Medical Oncology, Shanghai Pulmonary Hospital \& \\ Thoracic Cancer Institute, Tongji University School of Medicine, No. 507, Zheng Min Road, Shanghai 200433, China. \\ Email: caicunzhou_dr@163.com; tonyjiangdr@163.com.
}

Submitted Oct 22, 2019. Accepted for publication Nov 05, 2019.

doi: $10.21037 / \mathrm{atm} .2019 .11 .52$

View this article at: http://dx.doi.org/10.21037/atm.2019.11.52

Immune checkpoint inhibitors (ICIs) that target programmed cell death protein 1 (PD-1) and its ligand (PDL1), or cytotoxic T lymphocyte antigen-4 (CTLA-4) elicit durable antitumor responses in multiple cancer types. Yet, only a minority of patients could derive clinical benefit (1). Understanding genomic correlates of response to ICIs could lay a foundation for the development of novel biomarkers and treatment to further enhance the therapeutic benefit $(2,3)$. Previous studies demonstrated that genetic alterations of KEAP1 would dysregulate oxidative stress pathway, resulting in oncogenesis and drug- and radio-resistance in different cancers $(4,5)$. Recently, some exploratory analyses with limited samples showed the association between KEAP1 mutations and clinical benefit to ICI. However, a comprehensive analysis of KEAP1 mutation frequency and their predictive significance for ICI treatment outcome in diverse cancers has not yet been investigated. Therefore, we conducted this pan-cancer analysis by using online database to systematically characterize the prevalence and predictive value of KEAP1 mutations across multiple cancer types.

All included patients and sequencing data were identified from the cBioPortal online database (https://www. cbioportal.org) (6). KEAP1 mutations were defined as all kinds of nonsynonymous mutations including missense, frame-shift, splice site, nonstop, nonsense, and translation start site changes. To evaluate the difference of tumor mutation burden (TMB) level between KEAP1 mutant and wild type groups, a subset generated from MSK-IMPACT cohort was selected to avoid the selection bias and ensure the TMB could be comparable (7). The six immune infiltrates abundances including $\mathrm{B}$ cells, $\mathrm{CD}^{+} \mathrm{T}$ cells, $\mathrm{CD} 8^{+}$ $\mathrm{T}$ cells, dendritic cells, macrophages and neutrophils were estimated by using a web server for comprehensive analysis of tumor-infiltrating immune cells, named TIMER (Tumor Immune Estimation Resource, https://cistrome.shinyapps. io/timer/) (8). Kaplan-Meier curves with log-rank tests were used to determine the survival difference.

We summarized all the relevant data in Figure 1. The prevalence of KEAP1 mutations in 40,167 patients with distinct cancer types was $2.7 \%$ (Figure $1 A$ ), with patients with non-small cell lung cancer (NSCLC) having the highest levels of KEAP1 mutations (15.8\%). Most of the alterations were missense mutations. The prevalence and spectrum of KEAP1 mutations were slightly different in early-stage (441/10,967, TCGA cohort; Figure S1A) versus advanced-stage cancers $(388 / 10,945$, MSK-IMPACT cohort; Figure $S 1 B$ ). In the MSK-IMPACT cohort (7), TMB of patients with KEAP1 mutations was significantly higher than in those without the mutations (10 vs. 4 mutations/ $\mathrm{Mb}, \mathrm{P}<0.0001$; Figure $1 B$ ). This was validated in the ICItreated cohort (Figure 1C) (9). Notably, cancers with KEAP1 missense mutations had the highest TMB level (Figure S2). Together, these findings reveal a high prevalence of KEAP1 mutations and its close relationship with TMB level across cancer types, suggesting that KEAP1 mutations should be considered as biomarkers when conducting ICI treatment.

Next, we surveyed the relationship between KEAP1 mutations and overall survival (OS) in both whole group and ICI-treated cohort. We firstly found that patients with KEAP1 mutations showed a significantly shorter OS 

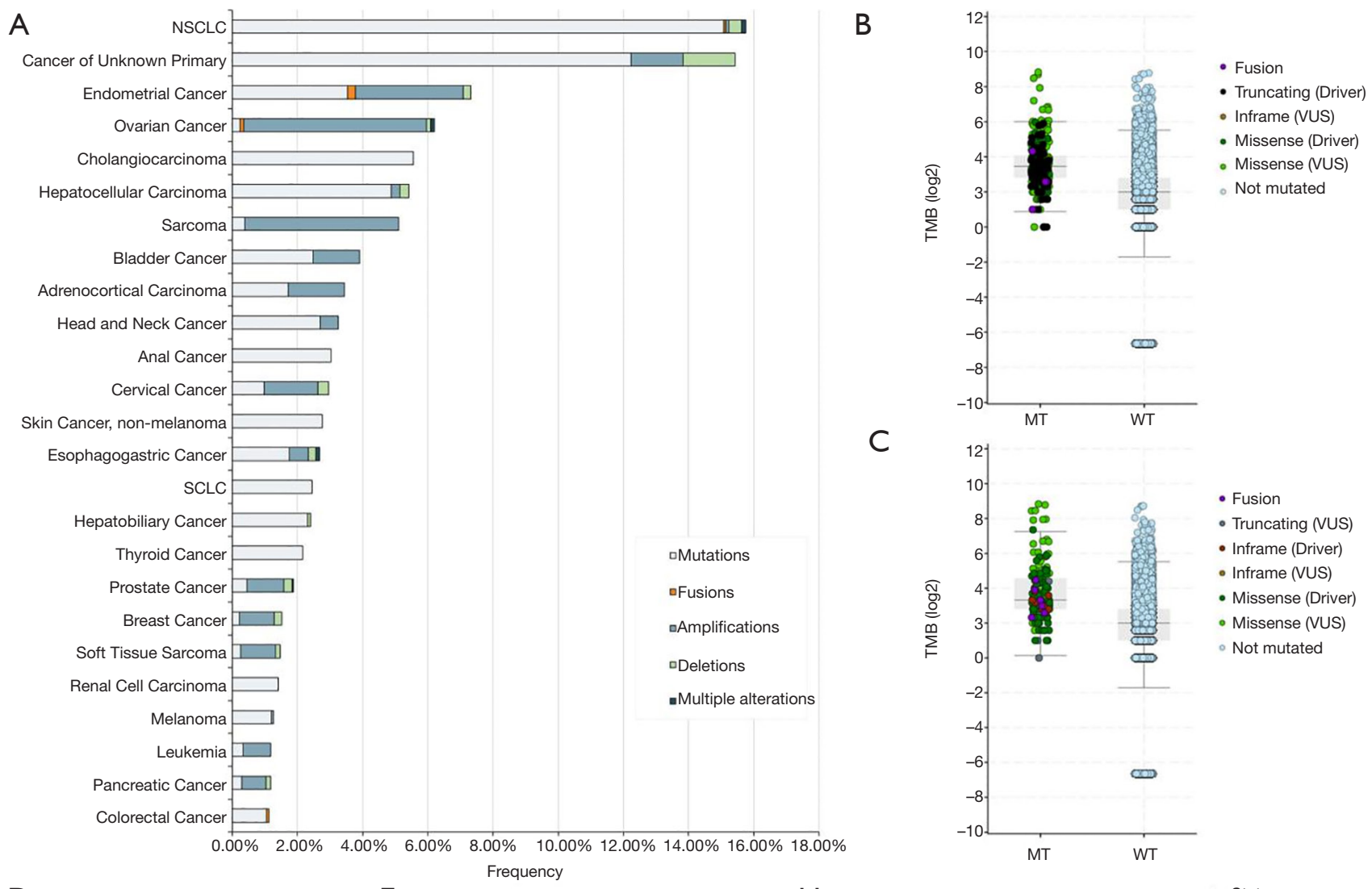

D
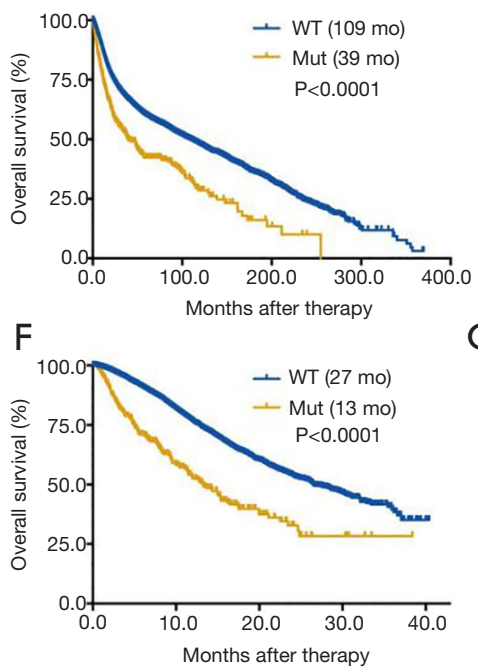

$E$

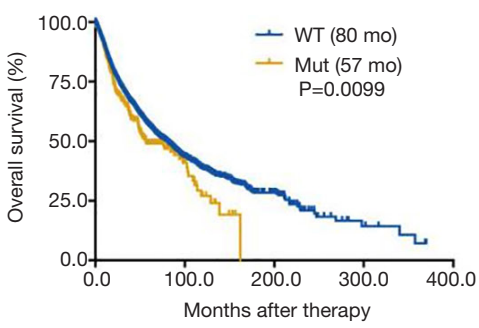

G

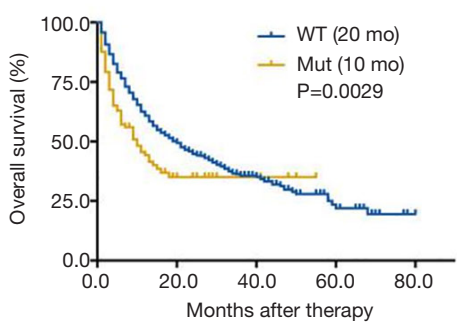

C

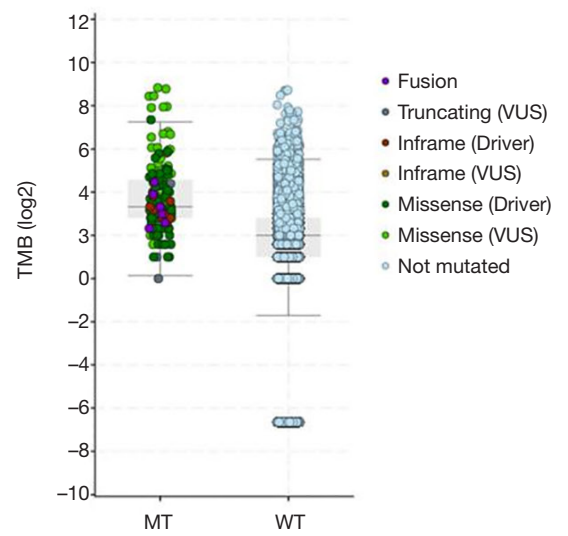

$\mathrm{H}$

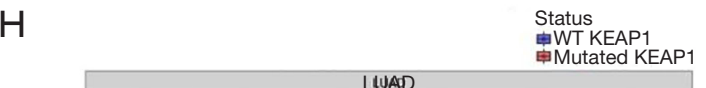

I

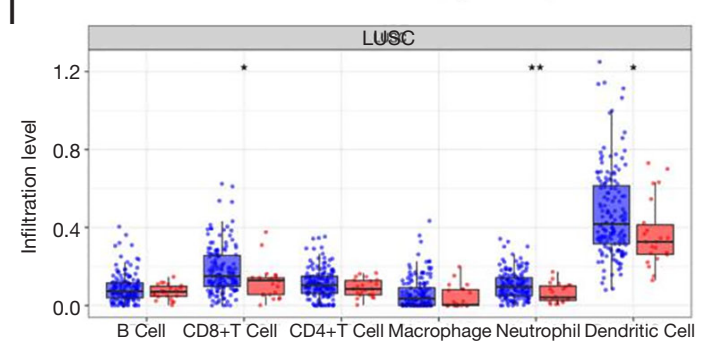

Figure 1 Pan-cancer analysis of KEAP1 mutations as biomarkers for immunotherapy outcomes. (A) Prevalence of KEAP1 mutations in different cancer types; (B) the association between TMB and KEAP1 mutations in MSK-IMPACT cohort; (C) the association between TMB and KEAP1 alterations in immune checkpoint inhibitors treatment cohort; (D) prognostic value of KEAP1 mutations in all cancers; (E) prognostic value of KEAP1 mutations in early-stage cancers (TCGA cohort); (F) prognostic value of KEAP1 mutations in advanced-stage cancers (MSK-IMPACT cohort); $(\mathrm{G})$ predictive value of KEAP1 mutations in patients received ICI therapy; $(\mathrm{H})$ the association between KEAP1 mutations and six immune infiltrates in lung adenocarcinoma; (I) the association between KEAP1 mutations and six immune infiltrates in lung squamous cell carcinoma. LUAD, lung adenocarcinoma; LUSC, lung squamous cell carcinoma; Mut, mutation; WT, wild type; TMB, tumor mutation burden; ICI, immune checkpoint inhibitor. 
(39 vs. 109 months, $\mathrm{P}<0.0001$; Figure $1 D$ ) than those without in whole populations. The prognostic value of KEAP1 mutations was also found in early-stage $(\mathrm{P}=0.0099$; Figure $1 E)$ and advanced-stage cancers $(\mathrm{P}<0.0001$; Figure $1 F)$. Although KEAP1 mutations were associated with marginally significantly shorter disease-free survival (DFS, 97 vs. 158 months, $\mathrm{P}=0.0677$; Figure S3A), it was associated with markedly inferior DFS in early-stage cancers $(\mathrm{P}=0.0009$; Figure $S 3 B)$. In the ICI treatment cohort (9), we identified 1,661 patients with different cancers receiving ICI therapy and 99 of them with KEAP1 mutations. Patients with KEAP1 mutations also had a substantially inferior OS of 10 vs. 20 months in the wildtype group ( $\mathrm{P}=0.0029$; Figure $1 G)$. Further investigation showed that only KEAP1 mutations could not predict OS in patients with microsatellite-stable (MSS) solid tumors (14 vs. 21 months, $\mathrm{P}=0.5619$; Figure S3C).

To unravel the potential mechanism of the predictive value of KEAP1 mutations for ICI treatment, we then investigated the association between KEAP1 mutations and immune landscape across multiple cancer types. We observed that these mutations were associated with significantly lower $\mathrm{CD}^{+} \mathrm{T}$ cells infiltrations in most of the cancer types including endometrial cancer, breast cancer, bladder cancer, colorectal cancer, lung adenocarcinoma (Figure 1H), lung squamous cell carcinoma (Figure 1I) and so on. Notably, patients with KEAP1-mutant lung adenocarcinoma had dramatically lower $\mathrm{CD} 8^{+} \mathrm{T}$ cells, neutrophils and dendritic cells infiltrations than those without, which was consistent with our recent publication (10). Of note, copy number variations (especially deep deletion or armlevel deletion) of KEAP1 were associated with substantially lower immune infiltrates in most cancer types including lung adenocarcinoma (Figure $S 4 A$ ) and lung squamous cell carcinoma (Figure $S 4 B$ ).

To our knowledge, this study firstly reported a high frequency of KEAP1 mutations in diverse cancers including lung cancer, endometrial cancer, hepatocellular carcinoma, head and neck cancer, bladder cancer, colorectal cancer, esophagogastric cancer, etc. and negative prognostic value of KEAP1 mutations for patients with different types of cancer. We also observed that KEAP1 mutations were a negative predictive biomarker and might be utilized to predict a survival benefit from ICI treatment across multiple cancers. Although KEAP1 mutations were correlated with significantly higher TMB level, they were also associated with significantly lower immune infiltrates especially $\mathrm{CD} 8^{+} \mathrm{T}$ cells, suggesting that tumor with these mutations could promote establishment of a coldtumor immune microenvironment. Considering the high prevalence of KEAP1 mutations, there is an urgent need for the development of rational and novel therapeutic. We are planning to initiate a prospective study to investigate the efficacy of PD-1 antibody plus vascular endothelial growth factor receptor tyrosine kinase inhibitors for patients with solid cancer and KEAP1 mutations. Collectively, our findings highlight the important value of KEAP1 alterations as pan-cancer predictive biomarkers for ICI treatment.

\section{Acknowledgment}

Funding: This study was supported in part by grants from the National Natural Science Foundation of China (No. 81672286, 81772467 and 81874036) and Medical Guidance Project of Shanghai Science and Technology Commission (No. 17411969200).

\section{Footnote}

Conflicts of Interest: The authors have no conflicts of interest to declare.

Ethical Statement: The authors are accountable for all aspects of the work in ensuring that questions related to the accuracy or integrity of any part of the work are appropriately investigated and resolved.

\section{References}

1. Sacher AG, Gandhi L. Biomarkers for the Clinical Use of PD-1/PD-L1 Inhibitors in Non-Small-Cell Lung Cancer: A Review. JAMA Oncol 2016;2:1217-22.

2. Keenan TE, Burke KP, Van Allen EM. Genomic correlates of response to immune checkpoint blockade. Nat Med 2019;25:389-402.

3. Binnewies M, Roberts EW, Kersten K, et al. Understanding the tumor immune microenvironment (TIME) for effective therapy. Nat Med 2018;24:541-50.

4. Jeong Y, Hoang NT, Lovejoy A, et al. Role of KEAP1/ NRF2 and TP53 Mutations in Lung Squamous Cell Carcinoma Development and Radiation Resistance. Cancer Discov 2017;7:86-101.

5. Tian Y, Wu K, Liu Q, et al. Modification of platinum sensitivity by KEAP1/NRF2 signals in non-small cell lung cancer. J Hematol Oncol 2016;9:83.

6. Gao J, Aksoy BA, Dogrusoz U, et al. Integrative analysis 
of complex cancer genomics and clinical profiles using the cBioPortal. Sci Signal 2013;6:pl1.

7. Zehir A, Benayed R, Shah RH, et al. Mutational landscape of metastatic cancer revealed from prospective clinical sequencing of 10,000 patients. Nat Med 2017;23:703-13.

8. Li T, Fan J, Wang B, et al. TIMER: A Web Server for Comprehensive Analysis of Tumor-Infiltrating Immune Cells. Cancer Res 2017;77:e108-10.

Cite this article as: Chen $\mathrm{X}, \mathrm{Su} \mathrm{C}$, Ren S, Zhou C, Jiang T. Pan-cancer analysis of KEAP1 mutations as biomarkers for immunotherapy outcomes. Ann Transl Med 2020;8(4):141. doi: 10.21037/atm.2019.11.52
9. Samstein RM, Lee CH, Shoushtari AN, et al. Tumor mutational load predicts survival after immunotherapy across multiple cancer types. Nat Genet 2019;51:202-6.

10. Jiang T, Shi J, Dong Z, et al. Genomic landscape and its correlations with tumor mutational burden, PD-L1 expression, and immune cells infiltration in Chinese lung squamous cell carcinoma. J Hematol Oncol 2019;12:75. 
A

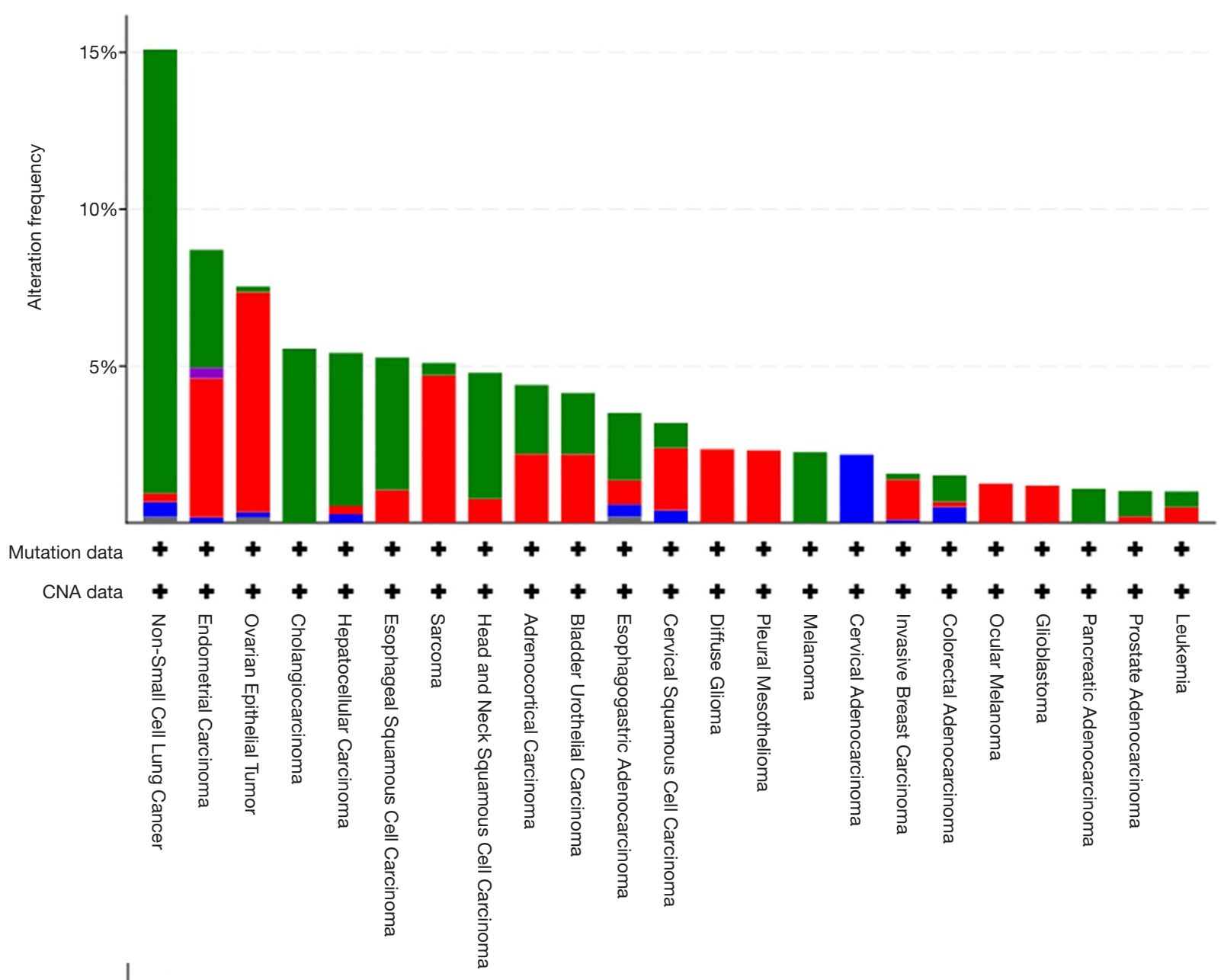

B

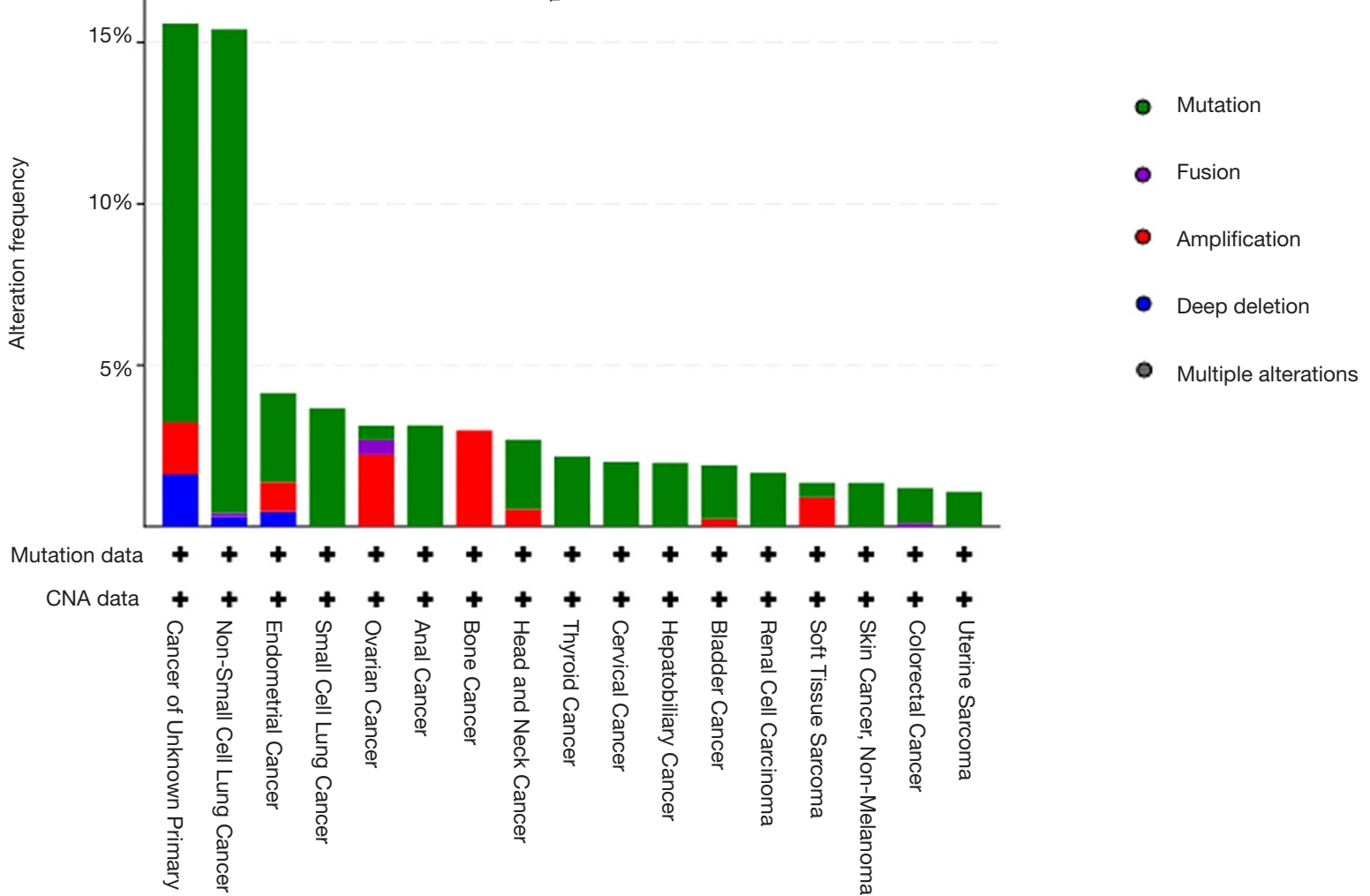

Figure S1 The frequency of KEAP1 mutations in early-stage cancer (A) and advanced-stage cancer (B). 

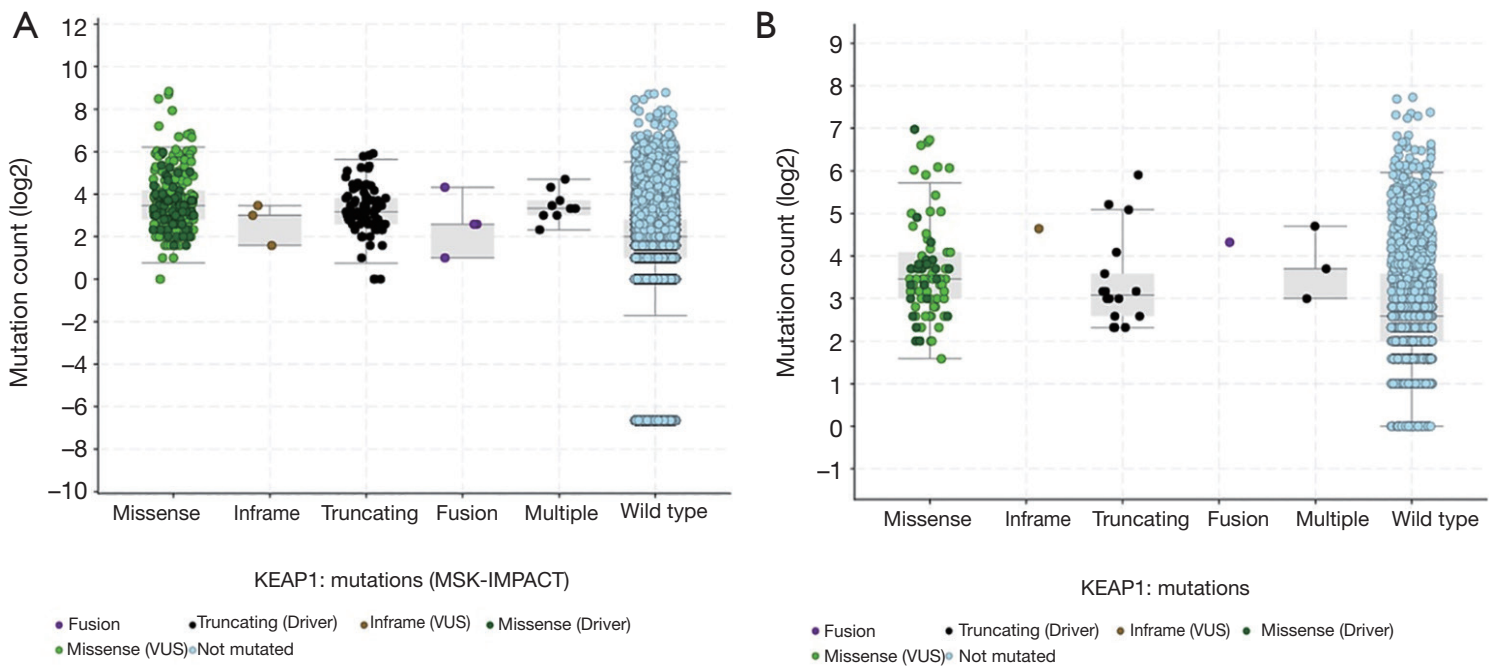

Figure S2 The association between TMB and KEAP1 mutations subtypes in MSK-IMPACT cohort (A) and immune checkpoint inhibitors treatment cohort (B). TMB, tumor mutation burden.
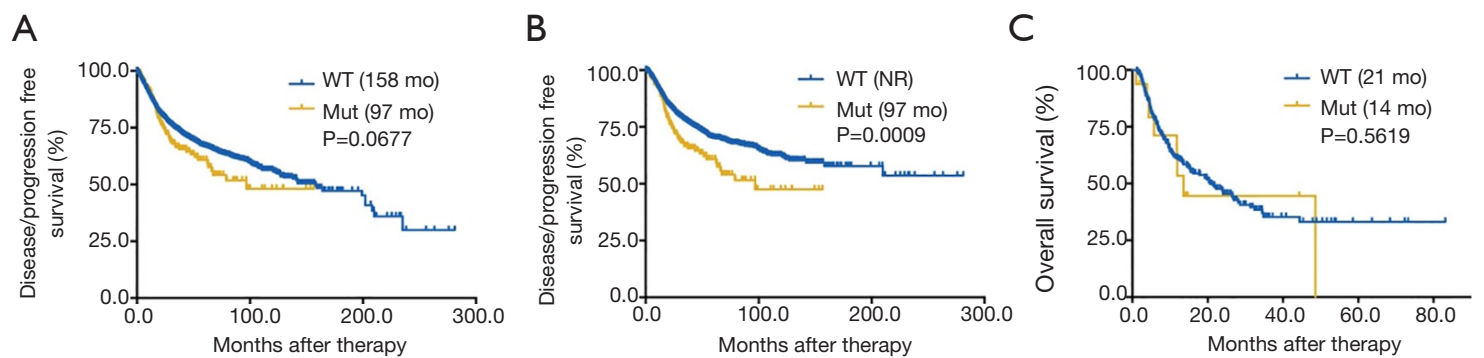

Figure S3 Predictive value of KEAP1 mutations in all cancers (A), in TCGA cohort (B) and in patients with MSS solid tumors (C). MSS, microsatellite-stable. 

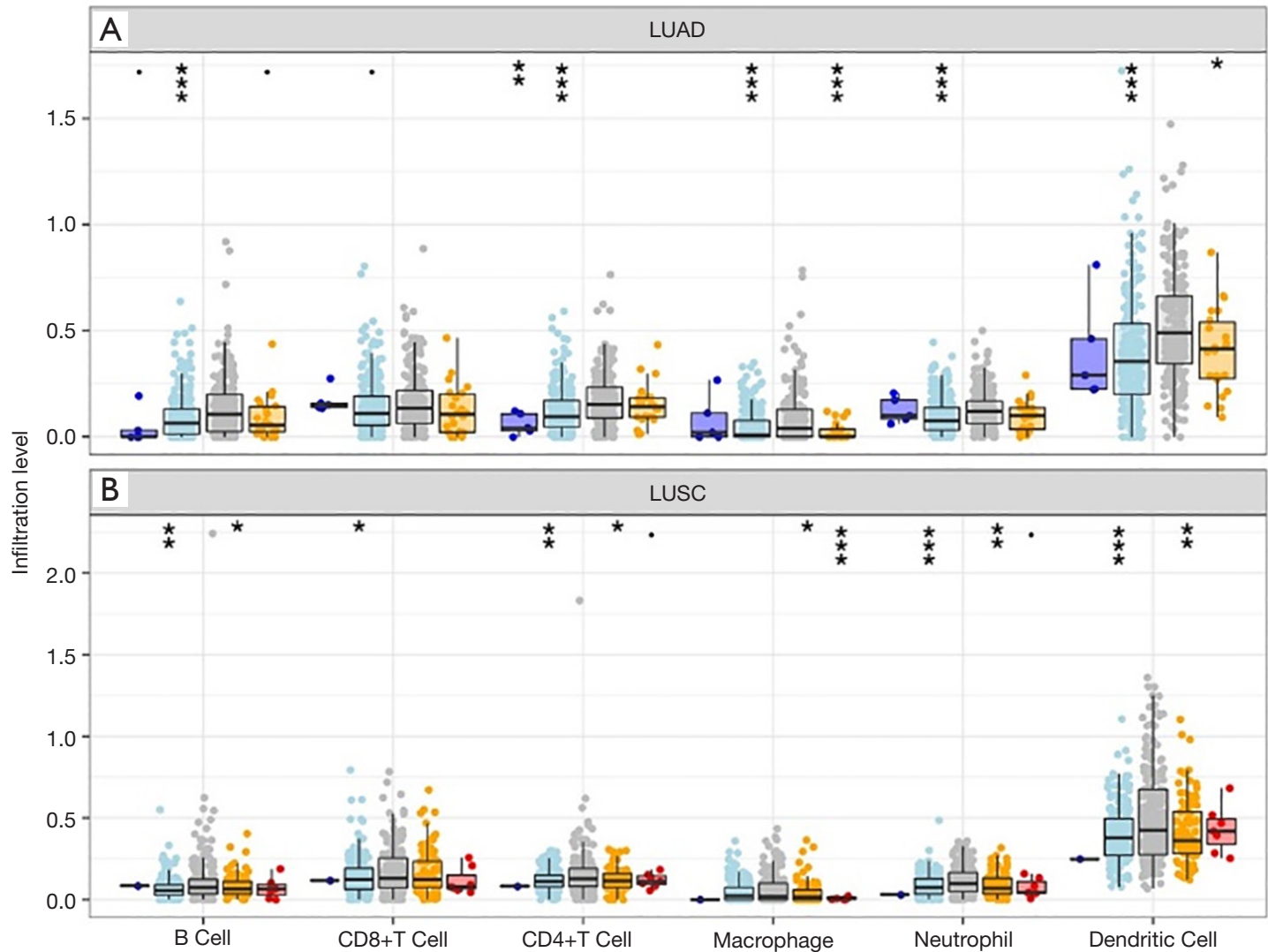

Copy number

由 Deep deletion

Arm-level deletion

由iploid/normal

舟 Arm-level gain

审 High amplication

Figure S4 The association between KEAP1 copy number variations and six immune infiltrates in lung adenocarcinoma (A) and lung squamous cell carcinoma (B). 